\title{
Perovskite photonic sources
}

\author{
Brandon R. Sutherland and Edward H. Sargent
}

Version Post-Print/Accepted Manuscript

Citation Sutherland, B. R., \& Sargent, E. H. (2016). Perovskite photonic sources.

(published version) Nature Photonics, 10(5), 295-302. doi:10.1038/nphoton.2016.62

Publisher's Statement The final published version of this article is available at Nature Photonics via https://dx.doi.org/10.1038/nphoton.2016.62.

How to cite TSpace items

Always cite the published version, so the author(s) will receive recognition through services that track citation counts, e.g. Scopus. If you need to cite the page number of the TSpace version (original manuscript or accepted manuscript) because you cannot access the published version, then cite the TSpace version in addition to the published version using the permanent URI (handle) found on the record page. 


\section{Perovskite photonic sources}

Brandon R. Sutherland, Edward H. Sargent*

Department of Electrical and Computer Engineering, University of Toronto, 10 King's College Road, Toronto, Ontario, M5S 3G4, Canada.

* Author to whom correspondence should be addressed.

Email: ted.sargent@utoronto.ca

The field of solution-processed semiconductors has made great strides; however, it has yet to enable electrically-driven lasers. To achieve this goal, improved materials are required that combine efficient ( $>50 \%$ quantum yield) radiative recombination under high injection, large and balanced charge carrier mobilities in excess of $1 \mathrm{~cm}^{2} \mathrm{~V}^{-1} \mathrm{~s}^{-1}$, and high absorption and gain coefficients exceeding $10^{4} \mathrm{~cm}^{-1}$. Solid-state perovskites are-in addition to galvanizing the field of solar electricity-showing great promise in photonic sources, and may be the answer to realizing solution-cast laser diodes. Here, we discuss the properties of perovskites that benefit light emission, review recent progress in perovskite electroluminescent diodes and optically pumped lasers, and examine the remaining challenges in achieving continuous-wave and electrically-driven lasing. 
Trihalide perovskite semiconductors are an emerging class of materials that have led to great strides in the performance of solution-processed optoelectronic devices. ${ }^{1-4}$ The bandgap is tunable via chemical composition, allowing it to be varied across visible and near-infrared wavelengths. ${ }^{5}$ High-quality perovskite thin films are formed at low temperature using inexpensive earth-abundant reagents. Their low Urbach energy (as small as $15 \mathrm{meV}),{ }^{6}$ minimal Stokes shift (less than $20 \mathrm{meV}$ ), ${ }^{7}$ high charge carrier mobility (in excess of $10 \mathrm{~cm}^{2} \mathrm{~V}^{-1} \mathrm{~s}^{-1}$ ), ${ }^{8}$ long diffusion length (up to $1 \mu \mathrm{m}$ and beyond), ${ }^{7,9-11}$ and low trap density (less than $10^{16} \mathrm{~cm}^{-3}$ ) ${ }^{12}$ have enabled rapid advances in the field of solution-processed solar cell technology.

Interest in perovskites soared once photovoltaic power conversion efficiencies ascended from $6.5 \%$ to $9.7 \%$ in $2012 .{ }^{13,14}$ These impressive efficiencies quickly put perovskites in contention with leading third-generation solar harvesting materials including dyes,${ }^{15}$ organic polymers,${ }^{16}$ colloidal quantum dots, ${ }^{17}$ and thin-film compound chalcogenides. ${ }^{18}$ By building upon pioneering advances in mesoscopic dye-sensitized solar cells, researchers increased the efficiency of perovskite photovoltaics dramatically over a span of less than three years. Breakthroughs in perovskite film formation, composition, and electrodes progressed efficiency to $10.9 \%,{ }^{19}$ $12 \%,{ }^{20,21} 15 \%,{ }^{22} 18.4 \%,{ }^{23} 20.1 \%,{ }^{24}$ and now to $21 \%$ certified. $^{25}$

Recently, researchers have explored new applications of this material, including in light-emitting diodes and in semiconductor optical amplifiers and lasers. We offer a critical evaluation of the prospects of perovskites in these important areas of application.

\section{Perovskite materials for light emission}

Perovskites (Figure 1a) possess the crystal structure $\mathrm{ABX}_{3}$, where $\mathrm{A}$ and $\mathrm{B}$ are cations jointly bound to X, an anion. Methylammonium (MA) lead trihalide perovskites have been the most 
intensively explored in optoelectronics to date. They have the chemical composition $\mathrm{CH}_{3} \mathrm{NH}_{3} \mathrm{PbX}_{\mathrm{n}} \mathrm{Y}_{3-\mathrm{n}}$, where $\mathrm{CH}_{3} \mathrm{NH}_{3}$ and $\mathrm{Pb}$ are cations and $\mathrm{X}$ and $\mathrm{Y}$ can be $\mathrm{I}, \mathrm{Br}$, or $\mathrm{Cl}$ anions. This perovskite is a hybrid inorganic-organic direct bandgap semiconductor spectrally tunable through compositional control of halides. The emission ranges from 390 to $790 \mathrm{~nm}$ (Figure 1b), and can be extended through cation substitution or blending. Methylammonium mixed with $\left(\mathrm{NH}_{2}\right)_{2} \mathrm{CH}^{+}$extends the emission wavelength to $820 \mathrm{~nm}$ and has led to the most recent photovoltaic performance breakthroughs. ${ }^{23,24} \mathrm{MASnX}_{3}$ perovskites have an emission wavelength tunable past $900 \mathrm{~nm}$ but are more sensitive to air, illumination, and external bias. ${ }^{26}$

Also extensively investigated in recent years are perovskites based on colloidal $\mathrm{CsPb}_{3}$ quantum dots. These perovskite nanoparticles offer a spectral range spanning 410-700 nm wavelength through both halide composition and quantum tuning. ${ }^{27}$ Their narrow photoluminescence spectra, coupled with their continuous spectral tunability, enable a pure colour distribution covering a range greater than the NTSC standard on a CIE chromaticity diagram (Figure 1c). Some studies have shown that these perovskites exhibit reduced blinking compared with other semiconductor quantum dot systems and that the exciton is less sensitive to dot size. ${ }^{28}$

Many of the underlying physical properties of perovskites that enabled the progress in solar cell efficiencies also enable enhancements in light emitting device performance. Perovskites possess a sharp optical absorption onset with $\alpha$, the absorption coefficient, exceeding $10^{4} \mathrm{~cm}^{-1}$ near the bandedge. ${ }^{6}$ A more strongly absorbing semiconductor can efficiently convert light to electrical current, and, correspondingly, can support higher material gain in a laser. This absorption coefficient surpasses even than that of GaAs, a leading semiconductor in high-performance commercial optoelectronic devices, including solar cells, light-emitting diodes, and lasers. 
The large mobilities in perovskites suggest promise in light emitting diodes (LEDs) that can achieve high brightness at low driving voltages. In lasing applications, the low Stokes shift reduces energy lost to heat during down-conversion of the pump. A clean bandgap with minimal charge-trapping defects increases the efficiency of band-to-band radiative recombination, central to the operation of light emitting devices. It has been shown that non-radiative pathways in perovskite thin films are slow and unfavourable, even at high pump intensities. Perovskites have demonstrated constant photoluminescence quantum yields near $70 \%$ at continuous-wave pump powers from 500-2000 $\mathrm{mW} \mathrm{cm}^{-2},{ }^{29}$ and an electroluminescence efficiency that continues to increase with injected current due to dominant bimolecular recombination. ${ }^{30}$

In addition to polycrystalline perovskite thin film fabrication, solution-based growth of large single crystal perovskites has recently been reported. ${ }^{11,10}$ These single crystals offer even lower bulk defect densities, of order $10^{9}-10^{11}$ deep trap states per cubic centimeter ${ }^{10,31}$ alongside diffusion lengths exceeding $5 \mu \mathrm{m},{ }^{10,11}$ and slower non-radiative pathways. ${ }^{32}$ To date, the dimensions of these crystals have made them ill-suited to form optoelectronic devices based on rectifying junctions. However, their applications as bulk semiconductor optical amplifiers and downconverters remain promising, and the prospect of substrate-controlled growth of patterned perovskite single crystals is a topic of intense research interest.

\section{Perovskite light emitting diodes}

Well before halide perovskites were utilized for solar light harvesting, they were studied for their electroluminescence (EL) properties. Early work by Saito and colleagues in 1994 demonstrated EL from the layered $\left(\mathrm{C}_{6} \mathrm{H}_{5} \mathrm{C}_{2} \mathrm{H}_{4} \mathrm{NH}_{3}\right)_{2} \mathrm{PbI}_{4}$ perovskite, although unoptimized fabrication meant that this was only achievable at cryogenic temperatures. ${ }^{33}$ 
The electronic and optical performance of solid-state perovskites is highly sensitive to the film formation method. The choice of solvent, annealing and drying conditions, precursor choice, ratio and concentration of reagents, and deposition sequence all determine the overall quality of the film. As a result of pioneering work on perovskite photovoltaics, there now exist multiple highly-tuned processing conditions for perovskite thin-films. Analysis of the detailed balance of emission and absorption connects excellent photovoltaic performance with excellent LED behaviour. ${ }^{34}$ The exceptional solar cell performance of perovskite photovoltaics, especially their high open-circuit voltage relative to their bandgap, predicts efficient operation as an LED. After the surge in perovskite photovoltaics led by advances in thin-film development, researchers once again began investigating the prospects of these excellent solar materials in LEDs.

A typical perovksite LED consists of an intrinsic active layer in a double-heterojunction structure (Figure 2a) with an n-type electron transport layer (ETL) and a p-type hole transport layer (HTL). Under forward bias, charge carriers are injected into a thin luminescent layer where they recombine radiatively, emitting light in all directions. Efficient LEDs utilize electrodes that readily inject carriers into the active region and prevent charges from passing through the device and quenching at contacts.

Early perovskite LEDs used $\mathrm{CH}_{3} \mathrm{NH}_{3} \mathrm{PbI}_{3}, \mathrm{CH}_{3} \mathrm{NH}_{3} \mathrm{PbBr}_{2} \mathrm{I}$, and $\mathrm{CH}_{3} \mathrm{NH}_{3} \mathrm{PbBr}_{3}$ emitting layers for near-infrared (NIR), red, and green emission respectively. ${ }^{30}$ In the NIR LED, the $\mathrm{CH}_{3} \mathrm{NH}_{3} \mathrm{PbI}_{3}$ active layer was formed between a titanium dioxide $\left(\mathrm{TiO}_{2}\right)$ ETL and a poly $\left(9,9^{\prime}-\right.$ dioctylfluorene) (F8) HTL. For the larger-bandgap green and red LEDs, PEDOT:PSS was used as an HTL and here F8 functioned as an ETL. The green LEDs reported in this work exhibited peak external quantum efficiency (EQE) of $0.1 \%$, and peak brightness of $364 \mathrm{~cd} \mathrm{~m}^{-2}$. Since this 
work, various perovskite LEDs utilizing different ETLs and HTLs have been reported (Figure $2 b)$.

The highest reported peak EQEs for perovskite LEDs are $3.5 \%^{35}$ and $8.5 \%{ }^{36}$ at near-infrared and green wavelengths respectively (Figure $2 \mathrm{c}$, left). The $8.5 \%$ value, achieved by optimizing the perovskite $\mathrm{MABr}: \mathrm{PbBr}_{2}$ precursor ratio and by pinning the perovskite grain size using molecular additives to confine the exciton, remains below the efficiencies (>20\%) demonstrated for both solution-processed polymer ${ }^{37}$ and colloidal quantum $\operatorname{dot}^{38}$ devices operating in the visible. The near-infrared efficiency more closely approaches the record demonstrated by CQD LEDs of $5.2 \%{ }^{39}$ The largest peak luminance in perovskite LEDs of $20,000 \mathrm{~cd} \mathrm{~m}^{-2}$ is the highest reported for solution-processed green LEDs and the peak radiance of $28 \mathrm{~W} \mathrm{sr}^{-1} \mathrm{~m}^{-2}$ is a record among solution-processed emitters in the NIR (Figure 2c, right). ${ }^{35}$

Recently, perovskite electroluminescence at blue wavelengths has been demonstrated, but with low efficiency. ${ }^{40,41}$ This results from suboptimal injecting electrodes and bandtailing observed in chloride perovskites. ${ }^{42,43}$ Perovskites, with complete tunability throughout the visible spectrum, are also a promising candidate for white-light LEDs. The first demonstrations of perovskite white-light emission have been achieved using a 2D layered perovskite with self-trapped emissive states, ${ }^{44}$ and a blended cation mixture of tunable perovskite nanocrystals embedded in a polymer matrix..$^{45}$

The inherent low exciton binding energy in perovskites - approximately a few $\mathrm{meV}$ - is a limiting factor in developing high efficiency LEDs. ${ }^{46}$ To promote radiative recombination, thin active regions are required to confine carriers spatially. Producing ultrathin perovskite films with complete substrate coverage has proved difficult and remains an ongoing challenge on the path to higher efficiency. Recent reports of stoichiometric-tuned reduced-dimensionality 
phenylethylammonium perovskites have been shown to possess higher exciton binding energies, and may show promise in light-emitting applications. ${ }^{47}$ Perovskite nanocrystals possess larger exciton binding energies than their bulk counterparts $;^{48}$ however, initial attempts at forming LEDs from these systems have resulted in lower efficiencies compared with bulk perovskites, primarily as a result of non-ideal surface passivation. ${ }^{49}$ The best performing perovskite nanocrystal LED exhibited an emission linewidth of $18 \mathrm{~nm}$ with peak EQE near $1 \%$ and peak brightness near $2000 \mathrm{~cd} \mathrm{~m}^{-2}$, highlighting the initial promise of these quantum confined materials for applications in light-emission. ${ }^{50}$

The reduced Auger loss and dominant radiative bimolecular recombination distinguishes perovskites among solution-processed materials, which are otherwise typically dominated by non-radiative losses at high carrier densities. Perovskites may therefore have distinct promise in the realization of high-intensity LEDs. However, more work needs to be done to understand the root cause of the diminishing performance at higher current densities. Some reports have associated this to be a result of sample degradation due to heat generation in the active layer, ${ }^{30}$ but others argue that non-radiative recombination indeed limits performance. ${ }^{51}$ Given that the photoluminescence quantum yield of $\mathrm{CH}_{3} \mathrm{NH}_{3} \mathrm{PbI}_{3}$ perovskite thin-films varies considerably based on sample preparation and processing conditions, it is reasonable that various perovskite LEDs exhibit different performance limitations under high-injection.

Continued work on film formation, tailoring electrodes for efficient injection, and the development of new perovskite active regions engineered to promote radiative recombination will continue to progress LED efficiency and promote higher brightness at lower injection currents. 


\section{Stimulated emission in perovskites}

Stimulated emission is the process by which photons induce the radiative relaxation of excitedstate electrons coherently (Figure 3a). Early evidence of stimulated emission in methylammonium lead trihalide perovskites was observed while studying the charge transfer dynamics of efficient perovskite solar cells using ultrafast pump-probe spectroscopy. ${ }^{14}$ Here, researchers observed negative total absorbance at energies just above the bandgap and identified it as a signature of stimulated emission, but then focused on studying the solar cell itself. As photovoltaic power conversion efficiencies soared, so too did the number of reports on perovskites for optical gain and lasing.

Optical amplification, given as gain per unit length in $\mathrm{cm}^{-1}$, can be studied by photoexciting the gain medium and measuring its spectral absorption in time. In perovskite thin-films, the optical gain has been reported to be as high as $3200 \pm 830 \mathrm{~cm}^{-1} .^{52}$ Values of this magnitude for a bulk material are comparable to single crystal GaAs, as expected given the high perovskite absorption

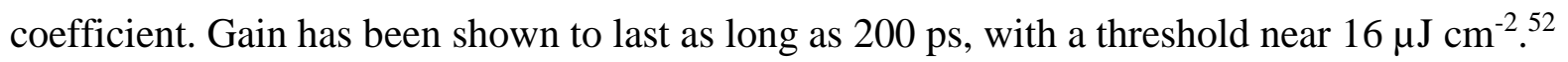

Gain as studied using ultrafast transient absorption gives an upper limit on the net gain that can be realized in an optical amplifier. When the modes emanating from the active material propagate, they suffer additional loss per unit length. Gain offset with propagation loss is reported as the net modal gain. It is measured using the variable stripe length technique. ${ }^{53}$ This method has been applied to perovskite thin films and net modal gains in the range of $66-250 \mathrm{~cm}^{-}$ ${ }^{1,43} 125 \mathrm{~cm}^{-1},{ }^{54}$ and $6-10 \mathrm{~cm}^{-1},{ }^{55}$ have been reported. Cesium lead halide perovskite nanocrystals have recently emerged as an efficient solution-processed gain media, exhibiting a net modal gain greater than $450 \pm 30 \mathrm{~cm}^{-1} .56$ 
Utilizing similar experimental conditions and varying the pump fluence instead of the stripe length while measuring the emitted spectral intensity profile yields the threshold for amplified spontaneous emission (ASE). Above the threshold for ASE, the luminescence spectrum greatly narrows and sharply increases in output intensity. Reported ASE thresholds range from $12 \mu \mathrm{J}$ $\mathrm{cm}^{-2}$ under $150 \mathrm{fs}$ excitation ${ }^{43}$ to $60 \mu \mathrm{J} \mathrm{cm}{ }^{-2}$ under 2 ns pumping conditions, ${ }^{54}$ and $7.6 \mu \mathrm{J} \mathrm{cm}^{-2}$ at pulse durations as long as $5 \mathrm{~ns}$ with the aid of a liquid crystal reflector. ${ }^{57}$ These early values are impressive, comparable to leading values for other solution-processed materials, including longstudied semiconductor polymers ${ }^{58}$ colloidal quantum dots, ${ }^{59}$ and colloidal nanoplates ${ }^{60}$ under similar excitation conditions.

Having demonstrated a net modal gain, perovskite researchers are exploring integration into amplification-exploiting devices: optically pumped lasers.

\section{Perovskite lasers}

Lasers require gain media capable of achieving population inversion, an optical cavity to provide feedback of coherent light, a method to outcouple this light, and net modal gain in the cavity configuration (Figure 3b).

The first report of a trihalide perovskite laser relied on a vertical surface emitting Fabry-Pérot cavity made by coating a distributed Bragg reflector with a perovskite thin-film, capped with a spacer layer and Au high reflector back mirror (Figure 3c). ${ }^{29}$ The device lased when excited using intense $400 \mathrm{ps}$ green laser light and produced lasing lines with a free spectral range of 9.5 THz. Since this work, there have been many new perovskite lasers utilizing both thin-films and large dimension single crystals. 
Most reported perovskite lasers have leveraged whispering gallery mode (WGM) cavities, where optical modes guided by internal reflection around the circumference of a circular or polygonal resonator provide feedback (Figure 3d). A spherical WGM laser enabled by conformal coating of perovskites with atomic layer deposition (ALD) onto glass microspheres leveraged a new materials processing strategy, termed perovskite ALD. ${ }^{54}$ The approach may interest the field of silicon photonics, which benefits from conformal strategies designed to integrate monolithically on structured substrates and cavities. Planar WGM mode lasers were also developed utilizing perovskite nanoplates. $\mathrm{CH}_{3} \mathrm{NH}_{3} \mathrm{PbI}_{3}$ polygonal plates grown via chemical vapour deposition exhibited a lasing threshold of $37 \mu \mathrm{J} \mathrm{cm}^{-2},{ }^{61}$ and solution-processed single crystal $\mathrm{CH}_{3} \mathrm{NH}_{3} \mathrm{PbBr}_{3}$ microdisks demonstrated a threshold of $3.6 \mu \mathrm{J} \mathrm{cm}{ }^{-2} .{ }^{62}$ Perovskite $\mathrm{CsPbX}_{3}$ nanocrystals have also demonstrated WGM lasing with a threshold of $5 \mu \mathrm{J} \mathrm{cm}^{-2} .{ }^{56}$ Taking advantage of the disorder in perovskite thin-films, random lasing from a $\mathrm{CH}_{3} \mathrm{NH}_{3} \mathrm{PbI}_{3}$ microcrystal network has also been demonstrated (Figure 3e) ${ }^{63,64}$ Recently, the first perovskite distributed feedback cavity laser was reported, with thresholds as low as $0.32 \mu \mathrm{J} \mathrm{cm}^{-2} .{ }^{65}$

Single crystal pristine and mixed iodine, bromine, and chlorine perovskite nanowires have been grown and used as Fabry-Pérot optical cavities for lasing (Figure 3f). ${ }^{32,66-68}$ Owing to the low trap state density and long Auger lifetimes, the resultant lasers demonstrate lasing thresholds as low as $220 \mathrm{~nJ} \mathrm{~cm}^{-2}$ with efficiencies approaching unity quantum yield, and quality factors of $3600 .{ }^{32}$ The latter two key figures of merit eclipse even the performance of epitaxial state-of-theart GaAs-AlGaAs nanowire lasers. The exact nature of the lasing cavity in these nanowires has been shown to be dependent on sample and pump positioning, and is a competition between the lengthwise Fabry-Pérot mode and the transverse whispering gallery mode. ${ }^{69}$ The stimulated emission thresholds in perovskite nanowire single crystals are lower than some of the best values 
reported in colloidal nanoplatelets, ${ }^{60}$ colloidal quantum dots, ${ }^{70}$ and organic polymers ${ }^{58}$ (Figure $3 g)$.

There has also been initial reports demonstrating more direct integration of perovskite gain media, including in amplifying waveguides ${ }^{71}$ and in outcoupling perovskite lasers with silver nanowire plasmonic waveguides. ${ }^{72}$

In addition to luminescent down-converters, LEDs, optical amplifiers, and lasers, perovskites have also been demonstrated to be efficient nonlinear emission sources. Both $\mathrm{MAPbBr}_{3}$ single crystal nanowires ${ }^{66}$ and $\mathrm{CsPbX}_{3}$ nanocrystals ${ }^{73}$ have exhibited multiphoton stimulated emission with thresholds of 674 and $2500 \mu \mathrm{J} \mathrm{cm}^{-2}$ respectively. $\mathrm{CsPbX}_{3}$ nanocrystals have also recently demonstrated room-temperature single photon emission with several benefits over traditional metal chalcogenide nonlinear emitters, such as an increased absorption cross-section, faster radiative recombination, and reduced dark exciton emission. ${ }^{74,75}$

\section{Challenges ahead}

Early organic conjugated polymer LEDs ${ }^{76}$ and lasers ${ }^{77}$ were demonstrated in the 1990 s, pioneering a field of solution-processed semiconductor light-emitters. Now, over two decades later, the field of light emission is still in search of a solution-processed material with efficient quantum yield at high pump intensity, a low threshold for stimulated emission, large balanced electron and hole mobilities, and a high damage threshold to facilitate electrically-driven lasing. Leading contenders include organic semiconductors, inorganic colloidal nanocrystals, and now perovskites. Organic semiconductors suffer from low damage thresholds, low carrier mobilities, and non-radiative losses from exciton-exciton annihilation at high carrier injection. ${ }^{78}$ Colloidal quantum dots have made impressive progress over the last two decades, and carry the promise of 
purely inorganic films with high damage thresholds; however, Auger recombination needs to be overcome to sustain population inversion for extended periods of time. ${ }^{59}$ Recently, colloidal quantum wells have shown promise with an indication of continuous-wave lasing and offer an interesting solution to the Auger recombination problem. ${ }^{79}$ However, their electrical properties are largely unexplored.

Perovskites have shown initial promise in their applications for light emission. Nonetheless, much remains for these materials to realize the ultimate goal of continuous-wave and, ideally, electrically-excited lasing.

Increasing the excitation pulse that produces sustained inversion and lasing, ultimately towards continuous-wave operation, is a first thrust. Perovskite thin-films possess a comparable Auger lifetime and stimulated emission threshold to that of colloidal nanoplatelets. Perovskite single crystals exhibit even further slowed Auger recombination and lower thresholds. This indicates promise for perovskite lasers exhibiting reduced heat generation under high power continuouswave optical pumping.

To date, perovskites have achieved room-temperature ASE with $5 \mathrm{~ns}, 100 \mathrm{~Hz}$ pump pulses, ${ }^{57}$ and with $100 \mathrm{~ns}$ pump pulses at temperatures up to $220 \mathrm{~K} .{ }^{80}$ Through an analysis of the feedback between ASE threshold state density and sample heating, it has been determined that the optical quality of the perovskite film and the substrate thermal conductivity are the two key parameters to further sustaining lasing over long durations of time. ${ }^{80}$ Recently, colloidal quantum dots have achieved microsecond lasing utilizing a high thermal conductivity substrate. ${ }^{81}$ Such strategies have yet to be employed for perovskite lasers, and processing conditions for high photoluminescence quantum-yield films have yet to be understood and reproduced consistently. Efforts towards continuous-wave lasing should develop strategies to effectively convey heat 
from the best perovskite active materials to raise the film ablation point above the threshold for stimulated emission under longer pulse durations.

To achieve electrically-excited lasing, several ingredients are necessary: a high quality gain medium with slow non-radiative decay pathways at the carrier density levels required for population inversion, large mobilities for both electrons and holes to minimize resistive heat loss, a large gain cross section at the lasing wavelength, and sharp band tails marked by a low Urbach energy. Perovskites have demonstrated all of these attributes, making them an exciting candidate for electrically-driven lasing.

A typical architecture for an edge-emitting electrically-pumped diode consists of a gain medium formed between thicker n-type ETL and p-type HTL cladding layers which have lower refractive indices. This semiconductor junction is forward biased, and a current density $\mathrm{J}$ is injected that is greater than the current threshold for population inversion, $\mathrm{J}_{\mathrm{th}}$. To form a laser, a cavity is required. A typical way to achieve this is by polishing one edge facet of the device, and roughening the other. Emitted light from the active layer becomes trapped in the higher refractive index gain medium, with the polished facet acting as an outcoupler, emitting laser light (Figure 4a).

The refractive index of hybrid organic-inorganic perovskites is typically greater than the organic ETLs and HTLs used in solution-processed LEDs. The thicknesses of these layers are typically of order 50-200 nm. In a laser diode, confining light in the active region and preventing leakage of the guided mode into the metal contacts is crucial. This requires suitably thick ( $>500 \mathrm{~nm}$ ) lower refractive index cladding layers that do not incur significant transport losses under high injection. The use of transparent top-contact conductive oxides may enable structures where both 
contacts have low refractive indices, permitting the use of more conventional thin ETL and HTL layers.

There have been some promising preliminary studies, such as achieving stimulated emission in the presence of electrically quenching contacts. ${ }^{43,82}$ The bulk defect density of perovskites is low, and these results suggest that the surface states of common ETL and HTLs do not diminish stimulated emission efficiency in thin-films.

As with continuous-wave optically pumped lasing, a principal challenge for electrical injection lasers is heat management. The large balanced electron and hole mobilities in high-performance perovskite thin-films, with respect to other contending technologies, correlate to reduced resistive heating under current injection. The projected temperature increase due to resistive heating, for a given mobility, $\mu$, and thermal conductivity, $\mathrm{K}$, was simulated (Figure $4 \mathrm{~b}$ ). A threshold current density of $1 \mathrm{kA} \mathrm{cm}^{-2}$, a typical value for compound semiconductor injection lasers, was used and was assumed to transit a $200 \mathrm{~nm}$ thick semiconductor film at an injected carrier density of $5 \times 10^{18} \mathrm{~cm}^{-3}$. Epitaxial single crystal semiconductors used in commercial injection lasers, such as GaAs and InP, offer both a large $\mathrm{K}^{83,84}$ and a high limiting $\mu^{85}$ (minimum of $\mu_{\text {electron }}$ and $\left.\mu_{\text {hole }}\right)$ and do not experience any significant resistive heating under large current flow. Colloidal quantum dots ${ }^{86}$ and organic semiconductors ${ }^{87}$ suffer from a small $\mathrm{K}$, are limited by low mobility for at least one carrier (typically electrons for organics ${ }^{88}$ and holes for CQDs ${ }^{89}$ ), resulting in a substantial temperature increase. Perovskites, while having similar K values to that of other solution-processed thin-films, ${ }^{90}$ possess large and balanced mobilities, resulting in reduced temperature increases due to resistive heat generation. These resistive heat losses underestimate the actual heat generation under current injection. All energy lost through non- 
radiative pathways will ultimately generate heat, in addition to limiting radiative efficiency, and heat management remains a critical point of control.

An essential requirement for applications of perovskite photonic sources will be stability under the relevant injection conditions. Light-emitting perovskite devices face many of the stability concerns seen in perovskite photovoltaic devices, perhaps even more stringently as a result of the high carrier densities typically generated in these devices. Some of the strategies of stabilizing the perovskite active medium while retaining high performance employed for photovoltaic devices hold promise, such as incorporating cesium into the lattice, ${ }^{91}$ varying the organic cation, ${ }^{92}$ using bifunctional alkylphosphonic crosslinking molecules, ${ }^{93}$ or with reduced dimensionality perovskites (which also have the added benefit of possessing a higher exciton binding energy). ${ }^{47}$ Using thermally stable carrier transport layers may also improve the stability of perovskite LEDs under high carrier injection. ${ }^{94}$ There have been some initial studies of stability in perovskite light-emitters, but there is still much to learn from the large body of work on stabilizing perovskite solar cells. Stimulated emission in $\mathrm{MAPbI}_{3}$ has been demonstrated over an initial 24 hour output study under continual pulsed excitation. ${ }^{43,54}$ Formamidinium lead halide perovskite nanowire lasers recently exhibited increased stability under continuous pulsed illumination in comparison with a $\mathrm{MAPbI}_{3}$ control. ${ }^{68}$ The lowest threshold perovskite lasers have also demonstrated no significant variation in lasing threshold after 4-6 months of storage in inert atmosphere. ${ }^{32}$

The toxicity of perovskite precursor materials is also an important point of consideration. All of the perovskites used for light-emitting applications to date, much like any high-performance perovskite solar cell, contain lead, a toxic substance. Implementing proper end-of-life management of any light-harvesting or light-emitting device using lead-based perovskites is 
critical to mitigate environmental impact. Incineration of perovskite films and subsequent

recovery of the $\mathrm{Pb}$ has been determined to be the most environmentally-sustainable protocol. ${ }^{95}$ Studies on $\mathrm{Pb}$-free perovskites for light emission are an encouraging yet unexplored avenue forward.

The photophysical properties of perovskites and the advancement of the solution-processed optical source field since their development distinguishes this new class of light emitters as a leading candidate for next-generation on-chip optical sources. Their promise for electricallydriven lasing and their prospects as a low-cost replacement for epitaxial III-V compound semiconductor gain media are bright.

\section{References}

1. Park, N.-G. Organometal perovskite light absorbers toward a $20 \%$ efficiency low-cost solidstate mesoscopic solar cell. J. Phys. Chem. Lett. 4, 2423-2429 (2013).

2. Kim, H.-S., Im, S. H. \& Park, N.-G. Organolead halide perovskite: New horizons in solar cell research. J. Phys. Chem. C 118, 5615-5625 (2014).

3. Green, M. A., Ho-Baillie, A. \& Snaith, H. J. The emergence of perovskite solar cells. Nat. Photonics 8, 506-514 (2014).

4. Stranks, S. D. \& Snaith, H. J. Metal-halide perovskites for photovoltaic and light-emitting devices. Nat. Nanotechnol. 10, 391-402 (2015).

5. Nobuaki Kitazawa, Watanabe, Y. \& Nakamura, Y. Optical properties of $\mathrm{CH}_{3} \mathrm{NH}_{3} \mathrm{PbX}_{3}(\mathrm{X}=$ halogen) and their mixed-halide crystals. J. Mater. Sci. 37, 3585-3587 (2002).

6. De Wolf, S. et al. Organometallic halide perovskites: sharp optical absorption edge and its relation to photovoltaic performance. J. Phys. Chem. Lett. 5, 1035-1039 (2014). 
7. Stranks, S. D. et al. Electron-hole diffusion lengths exceeding 1 micrometer in an organometal trihalide perovskite absorber. Science 342, 341-344 (2013).

8. Leijtens, T. et al. Electronic properties of meso-superstructured and planar organometal halide perovskite films: charge trapping, photodoping, and carrier Mobility. ACS Nano 8, $7147-7155$ (2014).

9. Xing, G. et al. Long-range balanced electron- and hole-transport lengths in organic-inorganic $\mathrm{CH}_{3} \mathrm{NH}_{3} \mathrm{PbI}_{3}$. Science 342, 344-347 (2013).

10. Shi, D. et al. Low trap-state density and long carrier diffusion in organolead trihalide perovskite single crystals. Science 347, 519-522 (2015).

11. Dong, Q. et al. Electron-hole diffusion lengths $>175 \mu \mathrm{m}$ in solution-grown $\mathrm{CH}_{3} \mathrm{NH}_{3} \mathrm{PbI}_{3}$ single crystals. Science 347, 967-970 (2015).

12. Stranks, S. D. et al. Recombination kinetics in organic-inorganic perovskites: excitons, free charge, and subgap states. Phys. Rev. Appl. 2, (2014).

13. Im, J.-H., Lee, C.-R., Lee, J.-W., Park, S.-W. \& Park, N.-G. 6.5\% efficient perovskite quantum-dot-sensitized solar cell. Nanoscale 3, 4088-4093 (2011).

14. Kim, H.-S. et al. Lead iodide perovskite sensitized all-solid-state submicron thin film mesoscopic solar cell with efficiency exceeding 9\%. Sci. Rep. 2, (2012).

15. Hardin, B. E., Snaith, H. J. \& McGehee, M. D. The renaissance of dye-sensitized solar cells. Nat. Photonics 6, 162-169 (2012).

16. Li, G., Zhu, R. \& Yang, Y. Polymer solar cells. Nat. Photonics 6, 153-161 (2012).

17. Kramer, I. J. \& Sargent, E. H. The architecture of colloidal quantum dot solar cells: materials to devices. Chem. Rev. 114, 863-882 (2013). 
18. Suryawanshi, M. P. et al. CZTS based thin film solar cells: a status review. Mater. Sci. Technol. 28, 98-109 (2013).

19. Lee, M. M., Teuscher, J., Miyasaka, T., Murakami, T. N. \& Snaith, H. J. Efficient hybrid solar cells based on meso-superstructured organometal halide perovskites. Science 338, 643647 (2012).

20. Heo, J. H. et al. Efficient inorganic-organic hybrid heterojunction solar cells containing perovskite compound and polymeric hole conductors. Nat. Photonics 7, 486-491 (2013).

21. Noh, J. H., Im, S. H., Heo, J. H., Mandal, T. N. \& Seok, S. I. Chemical management for colorful, efficient, and stable inorganic-organic hybrid nanostructured solar cells. Nano Lett. 13, 1764-1769 (2013).

22. Burschka, J. et al. Sequential deposition as a route to high-performance perovskite-sensitized solar cells. Nature 499, 316-319 (2013).

23. Jeon, N. J. et al. Compositional engineering of perovskite materials for high-performance solar cells. Nature 517, 476-480 (2015).

24. Yang, W. S. et al. High-performance photovoltaic perovskite layers fabricated through intramolecular exchange. Science 348, 1234-1237 (2015).

25. Research Cell Efficiency Records. (NREL, 2015). at <http://www.nrel.gov/ncpv/>

26. Hao, F., Stoumpos, C. C., Cao, D. H., Chang, R. P. H. \& Kanatzidis, M. G. Lead-free solidstate organic-inorganic halide perovskite solar cells. Nat. Photonics 8, 489-494 (2014).

27. Protesescu, L. et al. Nanocrystals of cesium lead halide perovskites $\left(\mathrm{CsPbX}_{3}, \mathrm{X}=\mathrm{Cl}, \mathrm{Br}\right.$, and I): novel optoelectronic materials showing bright emission with wide color gamut. Nano Lett. 15, 3692-3696 (2015). 
28. Swarnkar, A. et al. Colloidal $\mathrm{CsPbBr}_{3}$ perovskite nanocrystals: luminescence beyond traditional quantum dots. Angew. Chem. 127, 15644-15648 (2015).

29. Deschler, F. et al. High photoluminescence efficiency and optically pumped lasing in solution-processed mixed halide perovskite semiconductors. J. Phys. Chem. Lett. 5, 14211426 (2014).

30. Tan, Z.-K. et al. Bright light-emitting diodes based on organometal halide perovskite. Nat. Nanotechnol. 9, 687-692 (2014).

31. Adinolfi, V. et al. The in-gap electronic state spectrum of methylammonium lead iodide single crystal perovskites. (2016). doi:10.1002/adma.201505162

32. Zhu, H. et al. Lead halide perovskite nanowire lasers with low lasing thresholds and high quality factors. Nat. Mater. 14, 636-642 (2015).

33. Era, M., Morimoto, S., Tsutsui, T. \& Saito, S. Organic-inorganic heterostructure electroluminescent device using a layered perovskite semiconductor $\left(\mathrm{C}_{6} \mathrm{H}_{5} \mathrm{C}_{2} \mathrm{H}_{4} \mathrm{NH}_{3}\right)_{2} \mathrm{PbI}_{4}$. Appl. Phys. Lett. 65, 676-678 (1994).

34. Miller, O. D., Yablonovitch, E. \& Kurtz, S. R. Strong internal and external luminescence as solar cells approach the Shockley-Queisser limit. Photovolt. IEEE J. Of 2, 303-311 (2012).

35. Wang, J. et al. Interfacial control toward efficient and low-voltage perovskite light-emitting diodes. Adv. Mater. 27, 2311-2316 (2015).

36. Cho, H. et al. Overcoming the electroluminescence efficiency limitations of perovskite lightemitting diodes. Science 350, 1222-1225 (2015).

37. Aizawa, N. et al. Solution-processed multilayer small-molecule light-emitting devices with high-efficiency white-light emission. Nat. Commun. 5, (2014). 
38. Dai, X. et al. Solution-processed, high-performance light-emitting diodes based on quantum dots. Nature 515, 96-99 (2014).

39. Gong, X. et al. Highly efficient quantum dot near-infrared light-emitting diodes. Nat. Photonics (2016). doi:10.1038/NPHOTON.2016.11

40. Kumawat, N. K. et al. Band gap tuning of $\mathrm{CH}_{3} \mathrm{NH}_{3} \mathrm{~Pb}\left(\mathrm{Br}_{1-x} \mathrm{Cl}_{x}\right)_{3}$ hybrid perovskite for blue electroluminescence. ACS Appl. Mater. Interfaces 7, 13119-13124 (2015).

41. Sadhanala, A. et al. Blue-green color tunable solution processable organolead chloridebromide mixed halide perovskites for optoelectronic applications. Nano Lett. 15, 6095-6101 (2015).

42. Comin, R. et al. Structural, optical, and electronic studies of wide-bandgap lead halide perovskites. J Mater Chem C 3, 8839-8843 (2015).

43. Xing, G. et al. Low-temperature solution-processed wavelength-tunable perovskites for lasing. Nat. Mater. 13, 476-480 (2014).

44. Yangui, A. et al. Optical investigation of broadband white-light rmission in self-assembled organic-inorganic perovskite $\left(\mathrm{C}_{6} \mathrm{H}_{11} \mathrm{NH}_{3}\right)_{2} \mathrm{PbBr}_{4}$. J. Phys. Chem. C 119, 23638-23647 (2015).

45. Pathak, S. et al. Perovskite crystals for tunable white light emission. Chem. Mater. 27, 80668075 (2015).

46. Miyata, A. et al. Direct measurement of the exciton binding energy and effective masses for charge carriers in organic-inorganic tri-halide perovskites. Nat. Phys. 11, 582-587 (2015).

47. Quan, L. N. et al. Ligand-stabilized reduced-dimensionality perovskites. J. Am. Chem. Soc. (2016). doi:10.1021/jacs.5b11740 
48. Zheng, K. et al. Exciton binding energy and the nature of emissive states in organometal halide perovskites. J. Phys. Chem. Lett. 6, 2969-2975 (2015).

49. Song, J. et al. Quantum dot light-emitting diodes based on inorganic perovskite cesium lead halides $\left(\mathrm{CsPbX}_{3}\right)$. Adv. Mater. 27, $7162-7167$ (2015).

50. Zhang, X. et al. Enhancing the brightness of cesium lead halide perovskite nanocrystal based green light-emitting devices through the interface engineering with perfluorinated ionomer. Nano Lett. 16, 1415-1420 (2016).

51. Jaramillo-Quintero, O. A., Sanchez, R. S., Rincon, M. \& Mora-Sero, I. Bright visibleinfrared light emitting diodes based on hybrid halide perovskite with spiro-OMeTAD as a hole-injecting layer. J. Phys. Chem. Lett. 6, 1883-1890 (2015).

52. Sutherland, B. R. et al. Perovskite thin films via atomic layer deposition. Adv. Mater. 27, 5358 (2015).

53. Shaklee, K. L. Direct determination of optical gain in semiconductor crystals. Appl. Phys. Lett. 18, 475 (1971).

54. Sutherland, B. R., Hoogland, S., Adachi, M. M., Wong, C. T. O. \& Sargent, E. H. Conformal organohalide perovskites enable lasing on spherical resonators. ACS Nano 8, 10947-10952 (2014).

55. D’Innocenzo, V., Srimath Kandada, A. R., De Bastiani, M., Gandini, M. \& Petrozza, A. Tuning the light emission properties by band gap engineering in hybrid lead halide perovskite. J. Am. Chem. Soc. 136, 17730-17733 (2014).

56. Yakunin, S. et al. Low-threshold amplified spontaneous emission and lasing from colloidal nanocrystals of caesium lead halide perovskites. Nat. Commun. 6, 8056 (2015). 
57. Stranks, S. D. et al. Enhanced amplified spontaneous emission in perovskites using a flexible cholesteric liquid crystal reflector. Nano Lett. 15, 4935-4941 (2015).

58. Xia, R., Heliotis, G. \& Bradley, D. D. C. Fluorene-based polymer gain media for solid-state laser emission across the full visible spectrum. Appl. Phys. Lett. 82, 3599 (2003).

59. Dang, C. et al. Red, green and blue lasing enabled by single-exciton gain in colloidal quantum dot films. Nat. Nanotechnol. 7, 335-339 (2012).

60. She, C. et al. Low-threshold stimulated emission using colloidal quantum wells. Nano Lett. 14, 2772-2777 (2014).

61. Zhang, Q., Ha, S. T., Liu, X., Sum, T. C. \& Xiong, Q. Room-temperature near-infrared highQ perovskite whispering-gallery planar nanolasers. Nano Lett. 10, 687-692 (2014).

62. Liao, Q. et al. Perovskite microdisk microlasers self-assembled from solution. Adv. Mater. 27, 3405-3410 (2015).

63. Dhanker, R. et al. Random lasing in organo-lead halide perovskite microcrystal networks. Appl. Phys. Lett. 105, 151112 (2014).

64. Liu, S. et al. Random lasing actions in self-assembled perovskite nanoparticles. arXiv preprint (2015). arXiv:1512.07377

65. Saliba, M. et al. Structured organic-inorganic perovskite toward a distributed feedback laser. Adv. Mater. 28, 923-929 (02/16).

66. Gu, Z. et al. Two-photon pumped lead halide perovskite nanowire lasers. arXiv preprint (2015). arXiv:1510.03987v1

67. Xing, J. et al. Vapor phase synthesis of organometal halide perovskite nanowires for tunable room-temperature nanolasers. Nano Lett. 15, 4571-4577 (2015). 
68. Fu, Y. et al. Nanowire lasers of formamidinium lead halide perovskites and their stabilized alloys with improved stability. Nano Lett. 16, 1000-1008 (2016).

69. Wang, K. et al. Formation of single-mode laser in transverse plane of perovskite microwire via micromanipulation. Opt. Lett. 41, 555 (2016).

70. Park, Y.-S., Bae, W. K., Baker, T., Lim, J. \& Klimov, V. I. Effect of auger recombination on lasing in heterostructured quantum dots with engineered core/shell interfaces. Nano Lett. 15, 7319-7328 (2015).

71. Suárez, I., Juárez-Pérez, E. J., Bisquert, J., Mora-Seró, I. \& Martínez-Pastor, J. P. Polymer/perovskite amplifying waveguides for active hybrid silicon photonics. Adv. Mater. 27, 6157-6162 (2015).

72. Li, Y. J. et al. Output coupling of perovskite lasers from embedded nanoscale plasmonic waveguides. J. Am. Chem. Soc. (2016). doi:10.1021/jacs.5b12755

73. Wang, Y. et al. Nonlinear absorption and low-threshold multiphoton pumped stimulated emission from all-inorganic perovskite nanocrystals. Nano Lett. 16, 448-453 (2016).

74. Hu, F. et al. Superior optical properties of perovskite nanocrystals as single photon emitters. ACS Nano 9, 12410-12416 (2015).

75. Park, Y.-S., Guo, S., Makarov, N. S. \& Klimov, V. I. Room temperature single-photon emission from individual perovskite quantum dots. ACS Nano 9, 10386-10393 (2015).

76. Burroughes, J. H. et al. Light-emitting diodes based on conjugated polymers. Nature 347, 539-541 (1990).

77. Tessler, N., Denton, G. J. \& Friend, R. H. Lasing from conjugated-polymer microcavities. Nature 382, 695-697 (1996). 
78. Grivas, C. \& Pollnau, M. Organic solid-state integrated amplifiers and lasers. Laser Photonics Rev. 6, 419-462 (2012).

79. Grim, J. Q. et al. Continuous-wave biexciton lasing at room temperature using solutionprocessed quantum wells. Nat. Nanotechnol. 9, 891-895 (2014).

80. Cadelano, M. et al. Can trihalide lead perovskites support continuous wave lasing? Adv. Opt. Mater. 3, 1557-1564 (2015).

81. Adachi, M. M. et al. Microsecond-sustained lasing from colloidal quantum dot solids. Nat. Commun. 6, 8694 (2015).

82. Yuan, F. et al. Electric field-modulated amplified spontaneous emission in organo-lead halide perovskite $\mathrm{CH}_{3} \mathrm{NH}_{3} \mathrm{PbI}_{3}$. Appl. Phys. Lett. 107, 261106 (2015).

83. Voinigescu, S. High-Frequency Integrated Circuits. (Cambridge University Press, 2013).

84. Morkoc, H. Handbook of Nitride Semiconductors and Devices. 1, (Wiley-VCH, 2008).

85. Palankovski, V. \& Quay, R. Analysis and Simulation of Heterostructure Devices. (Springer Vienna, 2004).

86. Ong, W.-L., Rupich, S. M., Talapin, D. V., McGaughey, A. J. H. \& Malen, J. A. Surface chemistry mediates thermal transport in three-dimensional nanocrystal arrays. Nat. Mater. 12, 410-415 (2013).

87. Guo, Z. et al. Thermal conductivity of organic bulk heterojunction solar cells: an unusual binary mixing effect. Phys. Chem. Chem. Phys. 16, 26359-26364 (2014).

88. Köhler, A. Organic semiconductors: No more breaks for electrons. Nat. Mater. 11, 836-837 (2012). 
89. Yazdani, N., Bozyigit, D., Yarema, O., Yarema, M. \& Wood, V. Hole mobility in nanocrystal solids as a function of constituent nanocrystal size. J. Phys. Chem. Lett. 5, 35223527 (2014).

90. Pisoni, A. et al. Ultra-low thermal conductivity in organic-inorganic hybrid perovskite $\mathrm{CH}_{3} \mathrm{NH}_{3} \mathrm{PbI}_{3}$. J. Phys. Chem. Lett. 5, 2488-2492 (2014).

91. McMeekin, D. P. et al. A mixed-cation lead mixed-halide perovskite absorber for tandem solar cells. Science 351, 151-155 (2016).

92. Mei, A. et al. A hole-conductor-free, fully printable mesoscopic perovskite solar cell with high stability. Science 345, 295-298 (2014).

93. Li, X. et al. Improved performance and stability of perovskite solar cells by crystal crosslinking with alkylphosphonic acid $\omega$-ammonium chlorides. Nat. Chem. 7, 703-711 (2015).

94. Xu, J. et al. Crosslinked remote-doped hole-extracting contacts enhance stability under accelerated lifetime testing in perovskite solar cells. Adv. Mater. (2016). doi:10.1002/adma.201505630

95. Serrano-Lujan, L. et al. Tin- and lead-based perovskite solar cells under scrutiny: an environmental perspective. Adv. Energy Mater. 5, (2015).

96. Kumawat, N. K., Dey, A., Narasimhan, K. L. \& Kabra, D. Near infrared to visible electroluminescent diodes based on organometallic halide perovskites: structural and optical investigation. ACS Photonics 2, 349-354 (2015).

97. Li, G. et al. Efficient light-emitting diodes based on nanocrystalline perovskite in a dielectric polymer matrix. Nano Lett. 15, 2640-2644 (2015). 
98. Ling, Y. et al. Bright light-emitting diodes based on organometal halide perovskite nanoplatelets. Adv. Mater. 28, 305-311 (2016).

99. Yu, J. C., Kim, D. B., Jung, E. D., Lee, B. R. \& Song, M. H. High-performance perovskite light-emitting diodes via morphological control of perovskite films. Nanoscale (2016). doi:10.1039/C5NR05604G

100. Kim, Y.-H. et al. Multicolored organic/inorganic hybrid perovskite light-emitting diodes. Adv. Mater. 27, 1248-1254 (2015).

Figure 1. Perovskite structure and emission spectrum. a. Perovskites possess the general crystal structure $\mathrm{ABX}_{3}$. The most prevalent perovskite in optoelectronic devices is methylammonium lead trihalide, where $\mathrm{A}=\mathrm{CH}_{3} \mathrm{NH}_{3}, \mathrm{~B}=\mathrm{Pb}$, and $\mathrm{X}=\mathrm{Cl}$, $\mathrm{Br}$, or $\mathrm{I}$. b. Emission wavelength tunability of $\mathrm{CH}_{3} \mathrm{NH}_{3} \mathrm{PbX}_{n} \mathrm{Y}_{3-\mathrm{n}}$. The emission of the $\mathrm{MAPbX}_{n} \mathrm{Y}_{3-\mathrm{n}}$ perovskite is tunable from 390-790 nm wavelength. c. CIE chromaticity diagram. Perovskites have demonstrated color spaces covering a larger area than the National Television System Committee (NTSC) standard. ${ }^{27}$

Figure 2. Perovskite light emitting diodes. a. General operation of perovskite LEDs. b. Energy band alignment of methylammonium halide perovskites and the various ETLs and HTLs that have been used in reported perovskite LEDs. References: $\mathrm{PC}_{60} \mathrm{BM}$ (phenyl-C61-butyric acid methyl ester), ${ }^{40,96} \mathrm{ZnO}$ PEI (ZnO:polyethylenimine), ${ }^{35}$ PEDOT:PSS (poly(3,4ethylenedioxythiophene)-poly(styrenesulfonate)), ${ }^{30,40,96-99} \mathrm{~F} 8$ (poly(9,9'-dioctylfluorene), ${ }^{30,97}$ TPBI (1,3,5-tris(1-phenyl-1H-benzimidazol-2-yl)benzene), ${ }^{36,50,100}$ TPD (N,N'-bis(3- 
methylphenyl)-N,N'-diphenylbenzidine),${ }^{96}$ and TFB (poly(9,9-dioctyl-fluorene-co-N-(4-

butylphenyl)diphenylamine) ${ }^{35}$ c. EQE (left) and brightness/radiance (right) vs. current density for reported perovskite LEDs compared to leading polymer and CQD devices. Numbers correspond to references where valued were obtained. *This EQE is achieved from $10^{-4}$ to $10^{-1} \mathrm{~mA} \mathrm{~cm}^{-2}$.

Figure 3. Perovskite optical amplification. a. Schematic of ASE process. b. Optical amplification with a perovskite gain medium coupled with an optical cavity providing feedback enables lasing. c. Vertical surface emitting Fabry-Pérot laser cavity with a planar perovskite gain media. ${ }^{29}$ d. Whispering gallery mode cavity. ${ }^{54,56,61,62}$ e. Random lasing. ${ }^{63,64} \mathbf{f}$. Perovskite single crystal nanowire Fabry-Pérot cavities ${ }^{32,66-68}$ g. Stimulated emission threshold (Eth) vs. emission wavelength. Numbers correspond to references where valued were obtained.

Figure 4. Electrically-injected lasing and resistive heating under current. a. Electricallydriven laser schematic. Lasing is achieved when the injected current $\mathrm{J}$ is greater than the stimulated emission threshold current $\mathbf{J}_{\mathrm{th}}$. b. The resistive heating of a $200 \mathrm{~nm}$ semiconductor thin film with a carrier density of $5 \times 10^{18} \mathrm{~cm}^{-3}$ under $1 \mathrm{kA} \mathrm{cm}^{-2}$ current injection. Circles provide a general estimate of the limiting mobility in various semiconductors. 